\title{
A gradient projection method for solving split equality and split feasibility problems in Hilbert spaces
}

\section{Phan Tu Vuong, Jean Jacques Strodiot \& Van Hien Nguyen}

To cite this article: Phan Tu Vuong, Jean Jacques Strodiot \& Van Hien Nguyen (2015) A gradient projection method for solving split equality and split feasibility problems in Hilbert spaces, Optimization, 64:11, 2321-2341, DOI: 10.1080/02331934.2014.967237

To link to this article: http://dx.doi.org/10.1080/02331934.2014.967237

册Published online: 09 Oct 2014.

Submit your article to this journal $₫$

Џll Article views: 151

Q View related articles $\sqsubset$

View Crossmark data ¿ 


\title{
A gradient projection method for solving split equality and split feasibility problems in Hilbert spaces
}

\author{
Phan Tu Vuong ${ }^{\mathrm{ab}}$, Jean Jacques Strodiot ${ }^{\mathrm{ac} *}$ and Van Hien Nguyen ${ }^{\mathrm{ac}}$ \\ ${ }^{a}$ Institute for Computational Science and Technology (ICST), Ho Chi Minh City, Vietnam; \\ ${ }^{b}$ Department of Mathematics, University of Technical Education, Ho Chi Minh City, Vietnam; \\ ${ }^{c}$ Department of Mathematics, University of Namur, Namur, Belgium
}

(Received 13 September 2013; accepted 9 September 2014)

\begin{abstract}
In this paper, we first study in a Hilbertian framework the weak convergence of a general Gradient Projection Algorithm for minimizing a convex function of class $C^{1}$ over a convex constraint set. The way of selecting the stepsizes corresponds to the one used by López et al. for the particular case of the Split Feasibility Problem. This choice allows us to avoid the computation of operator norms. Afterwards, a relaxed version of the Gradient Projection Algorithm is considered where the feasible set is approximated by half-spaces making the projections explicit. Finally, to get the strong convergence, each step of the general Gradient Projection Method is combined with a viscosity step. This is done by adapting Halpern's algorithm to our problem. The general scheme is then applied to the Split Equality Problem, and also to the Split Feasibility Problem.
\end{abstract}

Keywords: gradient projection method; split equality problem; split feasibility problem; relaxed algorithms; strong convergence

AMS Subject Classifications: 65K05; 90C25; 47H10

\section{Introduction}

Let $H_{1}, H_{2}$ and $H_{3}$ be three infinite-dimensional real Hilbert spaces. The split equality problem recently studied by Byrne and Moudafi [1], and Moudafi [2,3] consists in

$(S E P) \quad$ Finding $x \in C$ and $y \in Q$ such that $A x=B y$

where $C$ and $Q$ are two nonempty closed convex subsets of $H_{1}$ and $H_{2}$, respectively, and $A: H_{1} \rightarrow H_{3}$ and $B: H_{2} \rightarrow H_{3}$ two bounded linear operators.

As mentioned in Moudafi [3], the interest of considering this problem is to cover many situations, for instance in decomposition methods for PDEs and in game theory. In decision sciences, this problem allows us to consider agents who interplay only via some components of their control variables. For further details, see Attouch et al. [4] and the references therein.

\footnotetext{
*Corresponding author. Email: jjstrodiot@ $@$ fundp.ac.be
} 
Here we assume that Problem $(S E P)$ has a solution $\left(x^{*}, y^{*}\right)$, i.e. the $S E P$ is consistent. So if we define

$$
f(z)=\frac{1}{2}\|A x-B y\|^{2} \quad \text { for every } z=(x, y) \in H_{1} \times H_{2}
$$

then $f$ is a nonnegative convex function of class $C^{1}$ on $H_{1} \times H_{2}$, and $z^{*}=\left(x^{*}, y^{*}\right)$ is a solution of the minimization problem

$$
\min f(z) \quad \text { subject to } z \in C \times Q
$$

that satisfies the property $f\left(z^{*}\right)=0$.

When the operator $B$ is the identity, the SEP becomes the split feasibility problem:

(SFP) Find $x \in C$ such that $A x \in Q$

where $C$ and $Q$ are two nonempty closed convex subsets of $H_{1}$ and $H_{2}$, respectively, and $A: H_{1} \rightarrow H_{2}$ is a bounded linear operator.

Censor and Elfving [5] first introduced in 1994 this Problem (SFP) for modelling inverse problems which arise in medical image reconstruction; see also Byrne [6] and Stark [7]. The Problem $(S F P)$ can also be applied to study intensity-modulated radiation therapy; see, for instance, Censor et al. [8]. Many well-known iterative algorithms for solving it were established; see Byrne [9], and see also the survey papers by Bauschke and Borwein [10] and by López et al. [11].

Here we assume that Problem $(S F P)$ has a solution $x^{*}$. So if we define

$$
f(x)=\frac{1}{2}\left\|\left(I-P_{Q}\right) A x\right\|^{2} \quad \text { for every } x \in C
$$

then $f$ is a nonnegative convex function of class $C^{1}$ on $H_{1}$, and $x^{*}$ is a solution of the minimization problem

$$
\min f(x) \quad \text { subject to } x \in C
$$

that satisfies the property $f\left(x^{*}\right)=0$. So in both cases we have to solve the minimization problem

$$
(P) \quad \min f(z) \quad \text { subject to } z \in S
$$

with the additional condition that the solution $z^{*} \in S$ satisfies $f\left(z^{*}\right)=0$ where $S$ is a nonempty closed convex subset of a Hilbert space $H$ and $f: H \rightarrow \mathbb{R}$ is a nonnegative convex function of class $C^{1}$. Many iterative methods have been investigated for solving the SFP. Among them, the most popular method is the Byrne CQ-algorithm [9], which generates a sequence $\left\{x_{k}\right\}$ by

$$
x_{k+1}=P_{C}\left(x_{k}-\lambda A^{*}\left(I-P_{Q}\right) A x_{k}\right) \quad \forall k \in \mathbb{N}
$$

where $x_{0} \in C$, the stepsize $\lambda$ is chosen in the interval $\left(0, \frac{2}{\|A\|^{2}}\right)$, and $A^{*}$ denotes the adjoint of the operator $A$. Recently, this method has been studied by Moudafi [12] when the sets $C$ and $Q$ are the fixed point sets of two quasi-nonexpansive operators, respectively.

As observed in López et al. [13], this method is nothing but the gradient projection method applied for minimizing on $C$ the function $f$ defined by

$$
f(x)=\frac{1}{2}\left\|\left(I-P_{Q}\right) A x\right\|^{2} \quad \text { for every } x \in C .
$$


Indeed, the iteration (1) can be rewritten as

$$
x_{k+1}=P_{C}\left(x_{k}-\gamma \nabla f\left(x_{k}\right)\right) \quad \forall k \in \mathbb{N} .
$$

Motivated by Byrne [9], Byrne and Moudafi proposed in [1] the following CQ-algorithm for solving the Split Equality Problem (SEP); see also Moudafi and Al-Shemas [14]. Given $\left(x_{0}, y_{0}\right) \in C \times Q$, compute successively the elements of the sequence $\left\{\left(x_{k}, y_{k}\right)\right\}$ by using the equalities

$$
\left\{\begin{array}{l}
x_{k+1}=P_{C}\left(x_{k}-\lambda_{k} A^{*}\left(A x_{k}-B y_{k}\right)\right) \\
y_{k+1}=P_{Q}\left(y_{k}+\lambda_{k} B^{*}\left(A x_{k}-B y_{k}\right)\right)
\end{array}\right.
$$

where the stepsize $\lambda_{k}$ is chosen in the interval $(a, b) \subset\left(0, \min \left\{\frac{1}{\|A\|^{2}}, \frac{1}{\|B\|^{2}}\right\}\right)$.

Let us mention that Moudafi [2] has also proposed a sequential version of (2) where the computation of $y_{k+1}$ is replaced by

$$
y_{k+1}=P_{Q}\left(y_{k}+\lambda_{k} B^{*}\left(A x_{k+1}-B y_{k}\right)\right) .
$$

If we define $f(z)=f(x, y)=\frac{1}{2}\|A x-B y\|^{2}$ for every $z=(x, y) \in H_{1} \times H_{2}$, it is easy to see that the iteration (2) can be rewritten as

$$
\left\{\begin{array}{l}
x_{k+1}=P_{C}\left(x_{k}-\lambda_{k} \nabla_{x} f\left(x_{k}, y_{k}\right)\right) \\
y_{k+1}=P_{Q}\left(y_{k}-\lambda_{k} \nabla_{y} f\left(x_{k}, y_{k}\right)\right)
\end{array}\right.
$$

Note that for computing $\lambda$ or $\lambda_{k}$ both iterative methods (1) and (2) need the a priori knowledge of the norm (or at least an estimate of the norm) of the bounded linear operators $A$ and $B$, which is, in general, not an easy work (see, for instance, Byrne [9] and López et al. [13]). To overcome this difficulty, an alternative way is to construct another stepsize that is independent of operator norms. A first attempt was made by Yang [15] who considered the following stepsize $\lambda_{k}=\rho_{k} /\left\|\nabla f\left(x_{k}\right)\right\|$ where $\left\{\rho_{k}\right\}$ is a sequence of positive real numbers such that

$$
\sum_{k=0}^{\infty} \rho_{k}=\infty \text { and } \sum_{k=0}^{\infty} \rho_{k}^{2}<\infty .
$$

Unfortunately, in order to obtain the convergence of the corresponding method, other conditions must be imposed on the step sequence $\left\{\lambda_{k}\right\}$ excluding some important cases (see López et al. [13] for more details). Recently, the authors in [13] modified the computation of $\lambda_{k}$ to remove the additional conditions imposed by Yang [15] in the CQ-algorithm for solving $(S F P)$. They proposed to use the sequence $\left\{x_{k}\right\}$ defined by

$$
x_{k+1}=P_{C}\left(x_{k}-\lambda_{k} A^{*}\left(I-P_{Q}\right) A x_{k}\right) \quad \forall k \in \mathbb{N}
$$

where $x_{0} \in C$ is given, and the stepsize $\lambda_{k}$ is chosen as

$$
\lambda_{k}=\frac{\rho_{k} f\left(x_{k}\right)}{\left\|\nabla f\left(x_{k}\right)\right\|^{2}}
$$

with $f(x)=\frac{1}{2}\left\|\left(I-P_{Q}\right) A x\right\|^{2}$ and $\rho_{k} \in(0,4)$. It was proved in ([13], Theorem 3.5) that if $\inf _{k} \rho_{k}\left(4-\rho_{k}\right)>0$, then the sequence $\left\{x_{k}\right\}$ generated by (4) with the step given by (5) converges weakly to a solution $x^{*}$ of $(S F P)$. The reader is referred to Polyak's book [16] for more details about the Gradient Projection Algorithm. 
In this paper, motivated by the work of López et al. [13], we first study the convergence of a general Gradient Projection Algorithm for minimizing a convex function $f$ of class $C^{1}$ over a convex constraint set $S$ using the stepsize $\lambda_{k}$ given by (5). Then we apply this general result first to the Split Equality Problem (SEP), and then after to the Split Feasibility Problem $(S F P)$. Doing so, we recover the convergence results of López et al. [13], and we obtain a new method for solving the Split Equality Problem (SEP) without the knowledge of operator norms $\|A\|$ and $\|B\|$.

When $C$ and $Q$ are the level sets associated with convex functions, the computation of the projections onto $C$ and $Q$ may be time-consuming. In such cases, many authors replaced the projections $P_{C}$ and $P_{Q}$ used in the previous iterative schemes by projections onto halfspaces containing the convex sets $C$ and $Q$, respectively. In that case, the projections become explicit. Let us mention here the papers by Byrne and Moudafi [1] and Moudafi [3] for Problem (SEP), and by López et al. [13], Xu [17], and Yang [18] for Problem (SFP).

In this paper, we consider a relaxed version of the Gradient Projection Algorithm. In this version, the feasible set is the level set of a convex function and the objective function $f$ is approximated at each iteration $k$ by a nonnegative convex function $f_{k}$ of class $C^{1}$. Conditions linking $f_{k}$ to $f$ are given to obtain the convergence of the sequence generated by the algorithm. This general scheme allows us to treat the Split Equality Problem (SEP) by taking $f_{k}=f$ for all $k$, and the Split Feasibility Problem (SFP) by choosing carefully the functions $f_{k}$.

Up to now, only weak convergence results have been discussed. However, it is well known that strongly convergent algorithms are of fundamental importance for solving infinite-dimensional problems. So in the last part of this paper we combine the general Gradient Projection Method with a viscosity procedure to get the strong convergence of the sequence of iterates generated by our scheme. This is done by adapting Halpern's algorithm [19] to our problem. As before, this general scheme is applied to the Split Equality Problem $(S E P)$ and the Split Feasibility Problem (SFP) giving each time the strong convergence of the generated sequences. Finally, let us mention the Tikhonov regularization method that was also used in Chen et al. [20] to obtain the strong convergence of the iterates.

After this paper has been submitted, the authors took note of a paper of Dong et al. [21] which was devoted to the Split Equality Problem. The difference between their algorithm and ours is in the choice of the step length that can be larger in our algorithm (see Section 3 below).

The paper is organized as follows. In the next Section, some preliminary results are recalled. In Section 3, a general Gradient Projection Algorithm is presented and the weak convergence of the sequence generated by this one is given. Then the particular cases of the Split Equality Problem and the Split Feasibility Problem are examined. In Section 4, we consider a relaxed version of the Gradient Projection Algorithm when the feasible sets $C$ and $Q$ are level sets of convex functions. The strong convergence of the method is obtained in Section 5 thanks to the incorporation of a viscosity step into the iterations. Finally, in Section 6, we give some concluding remarks.

\section{Preliminaries}

Let $H$ be a real Hilbert endowed with an inner product and its induced norm denoted $\langle\cdot, \cdot\rangle$ and $\|\cdot\|$, respectively. We write $x_{k} \rightarrow x\left(x_{k} \rightarrow x\right)$ to indicate that the sequence $\left\{x_{k}\right\}$ 
converges weakly (strongly) to $x$. Let $C$ be a nonempty closed convex subset of $H$. For each $x \in H$, there exists a unique point in $C$, denoted by $P_{C} x$, such that

$$
\left\|x-P_{C} x\right\| \leq\|x-y\| \quad \forall y \in C .
$$

It is known that $P_{C}$ is a nonexpansive mapping from $H$ onto $C$ and that for every $x \in H$, $u \in C$, the following property holds

$$
u=P_{C} x \Longleftrightarrow\langle x-u, v-u\rangle \leq 0 \quad \forall v \in C .
$$

On the other hand, a function $f: H \rightarrow \mathbb{R}$ is said to be weakly lower semi-continuous (l.s.c.) at $x$ if $x_{k} \rightarrow x$ implies

$$
f(x) \leq \liminf _{k \rightarrow \infty} f\left(x_{k}\right) .
$$

The function $f$ is said to be weakly 1.s.c. on $H$ if it is weakly l.s.c. at every point $x \in H$.

We also need the following lemma due to He and Yang [22]. This lemma is a new fundamental tool that generalizes a very well-known result due to $\mathrm{Xu}$ [23].

Lemm 2.1 Assume that $\left\{s_{k}\right\}$ is a sequence of nonnegative real numbers such that for all $k$

$$
\begin{aligned}
& s_{k+1} \leq\left(1-\alpha_{k}\right) s_{k}+\alpha_{k} \delta_{k}, \\
& s_{k+1} \leq s_{k}-\eta_{k}+\gamma_{k},
\end{aligned}
$$

where $\left\{\alpha_{k}\right\}$ is a sequence in $(0,1),\left\{\eta_{k}\right\}$ is a sequence of nonnegative real numbers, and $\left\{\delta_{k}\right\}$ and $\left\{\gamma_{k}\right\}$ are two sequences in $\mathbb{R}$ such that

(i) $\sum_{k=0}^{\infty} \alpha_{k}=\infty$,

(ii) $\lim _{k \rightarrow \infty} \gamma_{k}=0$,

(iii) $\lim _{k \rightarrow \infty} \eta_{n_{k}}=0$ implies that $\lim \sup _{k \rightarrow \infty} \delta_{n_{k}} \leq 0$ for any subsequence $\left\{n_{k}\right\}$ of $\{n\}$.

Then $\lim _{k \rightarrow \infty} s_{k}=0$.

\section{A gradient projection method}

In this section, we study a general Gradient Projection Method for minimizing a nonnegative convex function $f: H \rightarrow \mathbb{R}$ of class $C^{1}$ over a nonempty closed convex subset $S$ of $H$. We assume that this problem has a solution $z^{*}$ such that $f\left(z^{*}\right)=0$. We denote by $(P)$ this problem and by $\operatorname{SOL}(P)$ its solution set. Our aim is first to solve Problem $(P)$ and then to apply the obtained algorithm for solving the particular problems $(S E P)$ and $(S F P)$. For this purpose we introduce a gradient projection algorithm with a special stepsize that coincides with the one used by López et al. [13] when the problem $(S F P)$ is considered.

The Gradient Projection Algorithm we consider in this paper has the following form:

\section{ALGORITHM 1}

Step 1. Let $z_{0} \in S$. Set $k=0$.

Step 2. If $\nabla f\left(z_{k}\right)=0, \operatorname{STOP}\left(z_{k}\right.$ is a solution to $\left.(P)\right)$. Otherwise, go to Step 3 . 
Step 3. Compute the next iterate as follows

$$
z_{k+1}=P_{S}\left(z_{k}-\lambda_{k} \nabla f\left(z_{k}\right)\right)
$$

where the stepsize $\lambda_{k}$ is chosen in such a way that

$$
\lambda_{k}=\frac{\rho_{k} f\left(z_{k}\right)}{\left\|\nabla f\left(z_{k}\right)\right\|^{2}} \quad \text { with } \rho_{k} \in(0,4) .
$$

Step 4. Set $k \rightarrow k+1$ and go to Step 2 .

Since the function $f$ is convex, it is immediate that $z_{k} \in S$ is a minimum of $f$ over $S$ when $\nabla f\left(z_{k}\right)=0$. So in the sequel we assume that $\nabla f\left(z_{k}\right) \neq 0$ for all $k$. In order to prove the convergence of the sequence $\left\{z_{k}\right\}$ generated by Algorithm 1, we introduce the following assumptions on the sequences $\left\{z_{k}\right\},\left\{\lambda_{k}\right\}$, and $\left\{\rho_{k}\right\}$.

(C1) $\left\langle\nabla f\left(z_{k}\right), z_{k}-\bar{z}\right\rangle \geq 2 f\left(z_{k}\right)$ for all $k$ and every $\bar{z} \in \operatorname{SOL}(P)$.

(C2) $\lambda_{k} \geq \underline{\lambda}>0$ for all $k$.

(C3) $\inf _{k}\left[\rho_{k}\left(4-\rho_{k}\right)\right]>0$.

Since $f\left(z_{k}\right) \geq 0$ for all $k$, Condition $(\mathrm{C} 1)$ is related to the acute angle condition

$$
\langle\nabla f(z), z-\bar{z}\rangle \geq 0 \quad \text { for all feasible } z \neq \bar{z}
$$

that was introduced by Konnov [24] to prove the convergence of the gradient projection method. Thanks to this condition, we can write immediately for every $\bar{z} \in \operatorname{SOL}(P)$

$$
\begin{aligned}
\left\|z_{k+1}-\bar{z}\right\|^{2} & =\left\|P_{S}\left(z_{k}-\lambda_{k} \nabla f\left(z_{k}\right)\right)-P_{S}(\bar{z})\right\|^{2} \\
& \leq\left\|z_{k}-\lambda_{k} \nabla f\left(z_{k}\right)-\bar{z}\right\|^{2} \\
& \left.=\left\|z_{k}-\bar{z}\right\|^{2}-2 \lambda_{k}\left\langle\nabla f\left(z_{k}\right), z_{k}-\bar{z}\right\rangle+\lambda_{k}^{2} \| \nabla f\left(z_{k}\right)\right) \|^{2} \\
& \left.\leq\left\|z_{k}-\bar{z}\right\|^{2}-4 \lambda_{k} f\left(z_{k}\right)+\lambda_{k}^{2} \| \nabla f\left(z_{k}\right)\right) \|^{2} .
\end{aligned}
$$

Now we can choose $\lambda_{k}>0$ such that the right-hand side of (8) is minimized. This gives the following value for $\lambda_{k}$

$$
\lambda_{k}=\frac{2 f\left(z_{k}\right)}{\left\|\nabla f\left(z_{k}\right)\right\|^{2}} .
$$

However in our method, to have more flexibility, we choose

$$
\lambda_{k}=\frac{\rho_{k} f\left(z_{k}\right)}{\left\|\nabla f\left(z_{k}\right)\right\|^{2}} \quad \text { where } 0<\rho_{k}<4 .
$$

Let us also observe that the sequence $\left\{z_{k}\right\}$ generated by Algorithm 1 is contained in $S$.

Now we can prove the following weak convergence theorem for Algorithm 1.

Theorem 3.1 Assume that the solution set $\operatorname{SOL}(P)$ is nonempty. Let $\left\{z_{k}\right\},\left\{\lambda_{k}\right\}$, and $\left\{\rho_{k}\right\}$ be the sequences generated by Algorithm 1. If Conditions $(\mathrm{C} 1)-(\mathrm{C} 3)$ are satisfied, then

(a) For all $k$ and every $\bar{z} \in \operatorname{SOL}(P)$, the following inequality holds

$$
\left\|z_{k+1}-\bar{z}\right\|^{2} \leq\left\|z_{k}-\bar{z}\right\|^{2}-\rho_{k}\left(4-\rho_{k}\right) \frac{\left[f\left(z_{k}\right)\right]^{2}}{\left\|\nabla f\left(z_{k}\right)\right\|^{2}} ;
$$


(b) The sequence $\left\{z_{k}\right\}$ is bounded and $\sum_{k=0}^{\infty} \rho_{k}\left(4-\rho_{k}\right) \frac{\left[f\left(z_{k}\right)\right]^{2}}{\left\|\nabla f\left(z_{k}\right)\right\|^{2}}<\infty$;

(c) The sequence $\left\{z_{k}\right\}$ converges weakly to some $z^{*} \in \operatorname{SOL}(P)$ and the sequence $\left\{f\left(z_{k}\right)\right\}$ converges to 0 .

Proof The first statement is obtained immediately by replacing $\lambda_{k}$ by its value (9) in the inequality (8). Since $\rho_{k}\left(4-\rho_{k}\right)>0$, it follows from (a) that $\left\{\left\|z_{k}-\bar{z}\right\|\right\}$ is a decreasing sequence. As a consequence, the sequence $\left\{z_{k}\right\}$ is bounded. Furthermore, it follows immediately from (a) that for all $n$

$$
\sum_{k=0}^{n} \rho_{k}\left(4-\rho_{k}\right) \frac{\left[f\left(z_{k}\right)\right]^{2}}{\left\|\nabla f\left(z_{k}\right)\right\|^{2}} \leq\left\|z_{0}-\bar{z}\right\|^{2} .
$$

But this means that the second statement of (b) is proven. Now it remains to prove (c). In that purpose, first we observe that thanks to Condition (C3) and the convergence of the series given in (b), we can write that

$$
\frac{\left[f\left(z_{k}\right)\right]^{2}}{\left\|\nabla f\left(z_{k}\right)\right\|^{2}} \rightarrow 0 .
$$

Since $0<\rho_{k}<4$, we can also write, using the definition of $\lambda_{k}$ and Condition (C2), that

$$
\frac{4\left[f\left(z_{k}\right)\right]^{2}}{\left\|\nabla f\left(z_{k}\right)\right\|^{2}} \geq \frac{\rho_{k}\left[f\left(z_{k}\right)\right]^{2}}{\left\|\nabla f\left(z_{k}\right)\right\|^{2}}=\lambda_{k} f\left(z_{k}\right) \geq \underline{\lambda} f\left(z_{k}\right) \geq 0 .
$$

So we deduce that $f\left(z_{k}\right) \rightarrow 0$ and, since $\underline{\lambda}\left\|\nabla f\left(z_{k}\right)\right\|^{2} \leq 4 f\left(z_{k}\right)$, that $\left\|\nabla f\left(z_{k}\right)\right\| \rightarrow 0$.

Furthermore, using the nonexpansiveness of the projection, we obtain for all $k$ that

$$
\begin{aligned}
\left\|z_{k+1}-z_{k}\right\|^{2} & =\left\|P_{S}\left(z_{k}-\lambda_{k} \nabla f\left(z_{k}\right)\right)-P_{S}\left(z_{k}\right)\right\|^{2} \\
& \leq \lambda_{k}^{2}\left\|\nabla f\left(z_{k}\right)\right\|^{2} .
\end{aligned}
$$

Since $\left\|\nabla f\left(z_{k}\right)\right\| \rightarrow 0$, we can conclude that

$$
\frac{\left\|z_{k+1}-z_{k}\right\|^{2}}{\lambda_{k}^{2}} \rightarrow 0
$$

Let $z^{*}$ be a weak limit point of the bounded sequence $\left\{z_{k}\right\}$. Then there exists a subsequence of $\left\{z_{k}\right\}$, denoted again by $\left\{z_{k}\right\}$, that converges weakly to $z^{*}$. Since $z_{k+1}=P_{S}\left(z_{k}-\lambda_{k} \nabla f\left(z_{k}\right)\right)$, we have immediately that

$$
\frac{z_{k}-z_{k+1}}{\lambda_{k}}-\nabla f\left(z_{k}\right) \in N_{S}\left(z_{k+1}\right)
$$

where $N_{S}\left(z_{k+1}\right)$ denotes the normal cone to $S$ at $z_{k+1}$. Taking the limit in (11) as $k \rightarrow \infty$, we obtain that $0 \in N_{S}\left(z^{*}\right)$, and thus that $z^{*} \in S$.

On the other hand, the function $f$ being convex and strongly continuous is weakly l.s.c. and

$$
0 \leq f\left(z^{*}\right) \leq \liminf _{k \rightarrow \infty} f\left(z_{k}\right)=0
$$

because $f\left(z_{k}\right) \rightarrow 0$. Hence $f\left(z^{*}\right)=0$, and since $z^{*} \in S$, we can conclude that $z^{*} \in$ $\operatorname{SOL}(P)$.

It remains to prove that $z^{*}$ is the unique weak limit point of $\left\{z_{k}\right\}$. Suppose there exists another weak limit point $\tilde{z}$ of $\left\{z_{k}\right\}$. Replacing $z^{*}$ by $\tilde{z}$ in the previous part of the proof, we 
can deduce that $\tilde{z} \in \operatorname{SOL}(P)$. Furthermore thanks to (8) written with $\tilde{z}$ instead of $z^{*}$, we have that the sequences $\left\{\left\|z_{k}-z^{*}\right\|\right\}$ and $\left\{\left\|z_{k}-\tilde{z}\right\|\right\}$ are convergent.

On the other hand, since for all $k$,

$$
2\left\langle z_{k}, z^{*}-\tilde{z}\right\rangle=\left\|z_{k}-\tilde{z}\right\|^{2}-\left\|z_{k}-z^{*}\right\|^{2}+\left\|z^{*}\right\|^{2}-\|\tilde{z}\|^{2}
$$

we can conclude that the sequence $\left\{\left\langle z_{k}, z^{*}-\tilde{z}\right\rangle\right\}$ is also convergent. Let $l$ be its limit. Since $z^{*}$ and $\tilde{z}$ are the weak limits of two subsequences of $\left\{z_{k}\right\}$, we obtain that

$$
l=\left\langle z^{*}, z^{*}-\tilde{z}\right\rangle=\left\langle\tilde{z}, z^{*}-\tilde{z}\right\rangle,
$$

i.e. $\left\|z^{*}-\tilde{z}\right\|^{2}=0$. But this means that $z^{*}=\tilde{z}$ and thus that the whole sequence $\left\{z_{k}\right\}$ converges weakly to a vector $z^{*}$ in $\operatorname{SOL}(P)$.

Now we examine successively the particular problems SEP and SFP. Since, for Problem $(S E P), f(x, y)=\frac{1}{2}\|A x-B y\|^{2}$ for every $(x, y) \in C \times Q$, we have that

$$
\nabla f(x, y)=\left(A^{*}(A x-B y),-B^{*}(A x-B y)\right)
$$

for every $(x, y) \in C \times Q$, where $A^{*}$ and $B^{*}$ denote the adjoint operators of $A$ and $B$, respectively. In this case, Algorithm 1 can be rewritten as

\section{ALGORITHM 1-SEP}

Step 1. Let $\left(x_{0}, y_{0}\right) \in C \times Q$. Set $k=0$.

Step 2. If $A^{*}\left(A x_{k}-B y_{k}\right)=B^{*}\left(A x_{k}-B y_{k}\right)=0, \operatorname{STOP}\left(\left(x_{k}, y_{k}\right)\right.$ is a solution of Problem $(S E P))$. Otherwise, go to Step 3.

Step 3. Compute the next iterate $\left(x_{k+1}, y_{k+1}\right)$ as follows:

$$
\left\{\begin{array}{l}
x_{k+1}=P_{C}\left(x_{k}-\lambda_{k} A^{*}\left(A x_{k}-B y_{k}\right)\right) \\
y_{k+1}=P_{Q}\left(y_{k}+\lambda_{k} B^{*}\left(A x_{k}-B y_{k}\right)\right)
\end{array}\right.
$$

where the stepsize $\lambda_{k}$ is chosen in such a way that

$$
\lambda_{k}=\frac{\rho_{k}\left\|A x_{k}-B y_{k}\right\|^{2}}{2\left\|A^{*}\left(A x_{k}-B y_{k}\right)\right\|^{2}+2\left\|B^{*}\left(A x_{k}-B y_{k}\right)\right\|^{2}} \quad \text { with } \rho_{k} \in(0,4) .
$$

Step 4. Set $k \rightarrow k+1$ and go to Step 2 .

Recently, Dong et al. [21] have proposed a projection algorithm similar to Algorithm $1-S E P$ for solving Problem (SEP). The main difference with our algorithm is in the choice of the stepsize $\lambda_{k}$ in (12). If, at iteration $k$, we set

$$
a=\left\|A x_{k}-B y_{k}\right\|, \quad b=\left\|A^{*}\left(A x_{k}-B y_{k}\right)\right\|, \quad c=\left\|B^{*}\left(A x_{k}-B y_{k}\right)\right\|,
$$

then the stepsize used in [21] is chosen in the interval $\left(0, \min \left\{\frac{a^{2}}{b^{2}}, \frac{a^{2}}{c^{2}}\right\}\right)$ while our stepsize must be in the interval $\left(0, \frac{2 a^{2}}{b^{2}+c^{2}}\right)$. Since

$$
\min \left\{\frac{a^{2}}{b^{2}}, \frac{a^{2}}{c^{2}}\right\} \leq \frac{2 a^{2}}{b^{2}+c^{2}}
$$


the step $\lambda_{k}$ in our algorithm can be theoretically chosen larger than the one considered in [21].

When Problem (SFP) is considered, the function $f$ is defined for every $x \in C$ by $f(x)=\frac{1}{2}\left\|\left(I-P_{Q}\right) A x\right\|^{2}$. Then $\nabla f(x)=A^{*}\left(I-P_{Q}\right) A x$ for every $x \in C$. In this case, Algorithm 1 coincides with Algorithm 3.1 in López et al. [13] and takes the following form

\section{ALGORITHM 1-SFP}

Step 1. Let $x_{0} \in C$. Set $k=0$.

Step 2. If $\left(I-P_{Q}\right) A x_{k}=0, \operatorname{STOP}\left(x_{k}\right.$ is a solution of Problem $\left.(S F P)\right)$.

Otherwise, go to Step 3.

Step 3. Compute the next iterate as follows:

$$
x_{k+1}=P_{C}\left(x_{k}-\lambda_{k} A^{*}\left(I-P_{Q}\right) A x_{k}\right)
$$

where the stepsize $\lambda_{k}$ is chosen in such a way that

$$
\lambda_{k}=\frac{\rho_{k}\left\|\left(I-P_{Q}\right) A x_{k}\right\|^{2}}{\left.2 \| A^{*}\left(I-P_{Q}\right) A x_{k}\right) \|^{2}} \quad \text { with } \rho_{k} \in(0,4) .
$$

Step 4. Set $k \rightarrow k+1$ and go to Step 2 .

In the next two propositions we will prove the weak convergence of the sequences generated by Algorithms 1-SEP and 1-SFP to a solution of Problems (SEP) and (SFP), respectively. This is done by using Theorem 3.1 .

Proposition 3.2 Assume that the solution set of Problem (SEP) is nonempty and that the sequence $\left\{\rho_{k}\right\}$ satisfies Condition (C3). Then the sequence $\left\{\left(x_{k}, y_{k}\right)\right\}$ generated by Algorithm 1-SEP converges weakly to a solution of Problem (SE P). Moreover, the sequence $\left\{A x_{k}-B x_{k}\right\}$ converges strongly to 0 .

Proof In order to use Theorem 3.1, we have only to show that the sequences $\left\{z_{k}\right\}=$ $\left\{\left(x_{k}, y_{k}\right)\right\}$ and $\left\{\lambda_{k}\right\}$ satisfy Condition (C1) and Condition (C2), respectively. Let $\bar{z}=(\bar{x}, \bar{y})$ be a solution to Problem $(S E P)$ and let $z_{k}=\left(x_{k}, y_{k}\right) \in C \times Q$. Since $\nabla_{x} f\left(x_{k}, y_{k}\right)=$ $A^{*}\left(A x_{k}-B y_{k}\right)$, we easily obtain the following equalities

$$
\begin{aligned}
\left\langle\nabla_{x} f\left(x_{k}, y_{k}\right), x_{k}-\bar{x}\right\rangle & =\left\langle A^{*}\left(A x_{k}-B y_{k}\right), x_{k}-\bar{x}\right\rangle \\
& =\left\langle A x_{k}-B y_{k}, A x_{k}-A \bar{x}\right\rangle \\
& =\left\|A x_{k}-B y_{k}\right\|^{2}+\left\langle A x_{k}-B y_{k}, B y_{k}-A \bar{x}\right\rangle \\
& =2 f\left(x_{k}, y_{k}\right)+\left\langle A x_{k}-B y_{k}, B y_{k}-A \bar{x}\right\rangle .
\end{aligned}
$$

Similarly, since $\nabla_{y} f\left(x_{k}, y_{k}\right)=-B^{*}\left(A x_{k}-B y_{k}\right)$, we obtain

$$
\left\langle\nabla_{y} f\left(x_{k}, y_{k}\right), y_{k}-\bar{y}\right\rangle=2 f\left(x_{k}, y_{k}\right)-\left\langle A x_{k}-B y_{k}, A x_{k}-B \bar{y}\right\rangle .
$$

So Condition (C1) is satisfied because

$$
\begin{aligned}
\left\langle\nabla f\left(z_{k}\right), z_{k}-\bar{z}\right\rangle & =\left\langle\nabla_{x} f\left(x_{k}, y_{k}\right), x_{k}-\bar{x}\right\rangle+\left\langle\nabla_{y} f\left(x_{k}, y_{k}\right), y_{k}-\bar{y}\right\rangle \\
& =4 f\left(x_{k}, y_{k}\right)-\left\|A x_{k}-B y_{k}\right\|^{2} \\
& =2 f\left(x_{k}, y_{k}\right)=2 f\left(z_{k}\right) .
\end{aligned}
$$


On the other hand, since $\rho_{k} \geq \underline{\rho}>0$ (by Condition (C3)), the stepsize $\lambda_{k}$ satisfies the following relations for all $k$

$$
\begin{aligned}
\lambda_{k} & =\frac{\rho_{k} f\left(z_{k}\right)}{\left\|\nabla f\left(z_{k}\right)\right\|^{2}}=\frac{\rho_{k}\left\|A x_{k}-B y_{k}\right\|^{2}}{2\left\|A^{*}\left(A x_{k}-B y_{k}\right)\right\|^{2}+2\left\|B^{*}\left(A x_{k}-B y_{k}\right)\right\|^{2}} \\
& \geq \frac{\underline{\rho}\left\|A x_{k}-B y_{k}\right\|^{2}}{2\|A\|^{2}\left\|A x_{k}-B y_{k}\right\|^{2}+2\|B\|^{2}\left\|A x_{k}-B y_{k}\right\|^{2}}=\frac{\rho}{2} \frac{1}{\|A\|^{2}+\|B\|^{2}} .
\end{aligned}
$$

So Condition (C2) is satisfied. Finally, using Theorem 3.1, we obtain that $f\left(x_{k}, y_{k}\right) \rightarrow 0$, i.e. the sequence $\left\{A x_{k}-B x_{k}\right\}$ converges strongly to 0 .

For Problem (SFP) we can recover from Theorem 3.1 the following convergence result due to López et al. ([13], Theorem 3.5):

Proposition 3.3 Assume that the solution set of Problem (SF P) is nonempty and that the sequence $\left\{\rho_{k}\right\}$ satisfies Condition (C3). Then the sequence $\left\{x_{k}\right\}$ generated by Algorithm 1-SFP converges weakly to a solution of Problem (SF P). Moreover, the sequence $\{(I-$ $\left.\left.P_{Q}\right) A x_{k}\right\}$ converges strongly to 0 .

Proof In order to use Theorem 3.1, we have only to prove that the sequences $\left\{x_{k}\right\}$ and $\left\{\lambda_{k}\right\}$ satisfy Condition (C1) and Condition (C2), respectively. Let $\bar{x}$ be a solution to problem $(S F P)$ and let $x \in C$. Since $\nabla f(x)=A^{*}\left(I-P_{Q}\right) A x$, we can write

$$
\begin{aligned}
\langle\nabla f(x), x-\bar{x}\rangle & =\left\langle\left(I-P_{Q}\right) A x, A x-A \bar{x}\right\rangle \\
& =\left\langle\left(I-P_{Q}\right) A x, A x\right\rangle-\left\langle A x,\left(I-P_{Q}\right) A \bar{x}\right\rangle \\
& =\left\|\left(I-P_{Q}\right) A x\right\|^{2}=2 f(x)
\end{aligned}
$$

where we have used $P_{Q}(A \bar{x})=A \bar{x}$ and $\left(I-P_{Q}\right)\left(I-P_{Q}\right)=I-P_{Q}$. So Condition $(\mathrm{C} 1)$ is satisfied. On the other hand, since $\rho_{k} \geq \rho>0$, the stepsize $\lambda_{k}$ satisfies the following relations for all $k$

$$
\lambda_{k}=\frac{\rho_{k} f\left(x^{k}\right)}{\left\|\nabla f\left(x_{k}\right)\right\|^{2}}=\frac{\rho_{k}\left\|\left(I-P_{Q}\right) A x_{k}\right\|^{2}}{2\left\|A^{*}\left(I-P_{Q}\right) A x_{k}\right\|^{2}} \geq \frac{\underline{\rho}}{2\|A\|^{2}} .
$$

So Condition (C2) is satisfied. Finally, using Theorem 3.1, we obtain that $f\left(x_{k}\right) \rightarrow 0$, i.e. the sequence $\left\{\left(I-P_{Q}\right) A x_{k}\right\}$ converges strongly to 0 .

\section{A relaxed gradient projection method}

In Section 3, we studied a gradient projection method for solving Problem $(P)$. In that method it was supposed that the orthogonal projection onto the feasible set $S$ is easy to obtain. It was the case, for example, when $S$ is a ball, a half-space or a convex polyhedron. However, when the set $S$ is defined by means of convex inequalities, the computation of the projection onto $S$ is generally difficult. To overcome this difficulty, Fukushima [25] suggested to approximate the set $S$ by a sequence of half-spaces $\left\{S_{k}\right\}$ that contain it. Then the projection onto $S$ is replaced by projections onto the half-spaces $S_{k}$. This idea was followed by Yang [18] and Moudafi [3] for solving (SFP) and (SEP), respectively. Here we use the same strategy but applied to Problem $(P)$. As a consequence the sequence of points 
generated by the algorithm does not remain necessarily in the set $S$. Practically, we assume that the set $S$ is defined by

$$
S=\{z \in H \mid s(z) \leq 0\}
$$

where $s: H \rightarrow \mathbb{R}$ is a convex 1.s.c. (w.r.t. the strong topology) function defined on a real Hilbert space $H$. In this section, we also assume that the function $s$ is subdifferentiable on $H$ and that at each point $z$ some subgradient of $s$ at $z$ can be computed. So, if $\psi_{k} \in \partial s\left(z_{k}\right)$ (i.e. $\psi_{k}$ is a subgradient of $s$ calculated at $z_{k}$ ), the level set $S$ is approximated at $z_{k}$ by the half-space

$$
S_{k}=\left\{z \in H \mid s\left(z_{k}\right)+\left\langle\psi_{k}, z-z_{k}\right\rangle \leq 0\right\}
$$

(see, e.g. [22]). Then the projection $\bar{z}$ of a point $z \notin S_{k}$ onto $S_{k}$ is explicitly given by

$$
\bar{z}=z_{k}-\frac{s\left(z_{k}\right)}{\left\|\psi_{k}\right\|^{2}} \psi_{k} .
$$

Here we assume that the objective function $f: H \rightarrow \mathbb{R}$ of Problem $(P)$ is convex and 1.s.c. (in the strong topology). In order that our general model can solve both problems $(S E P)$ and $(S F P)$, we also consider a sequence $\left\{f_{k}\right\}$ of nonnegative convex functions of class $C^{1}$ defined on $H$ that approximate $f$ in the sense given below by Condition (C4). More precisely, we consider the following relaxed algorithm for solving Problem $(P)$.

\section{ALGORITHM 2}

Step 1. Let $z_{0} \in S$. Set $k=0$.

Step 2. If $\nabla f_{k}\left(z_{k}\right)=0, \operatorname{STOP}\left(z_{k}\right.$ is a solution to $\left.(P)\right)$. Otherwise, go to Step 3 .

Step 3. Compute $\psi_{k} \in \partial s\left(z_{k}\right)$ and the next iterate as follows

$$
z_{k+1}=P_{S_{k}}\left(z_{k}-\lambda_{k} \nabla f_{k}\left(z_{k}\right)\right)
$$

where the set $S_{k}=\left\{z \in H \mid s\left(z_{k}\right)+\left\langle\psi_{k}, z-z_{k}\right\rangle \leq 0\right\}$ and the stepsize $\lambda_{k}$ is chosen in such a way that

$$
\lambda_{k}=\frac{\rho_{k} f_{k}\left(z_{k}\right)}{\left\|\nabla f_{k}\left(z_{k}\right)\right\|^{2}} \quad \text { with } \rho_{k} \in(0,4) .
$$

Step 4. Set $k \rightarrow k+1$ and go to Step 2.

Let us observe that Algorithm 2 is implementable because the projections onto halfspaces $S_{k}$ are explicit (via the formula (15)). In the next theorem, we need to replace Condition $(\mathrm{C} 1)$ by the following condition

(C1a) $\left\langle\nabla f_{k}\left(z_{k}\right), z_{k}-\bar{z}\right\rangle \geq 2 f_{k}\left(z_{k}\right)$ for all $k$ and every $\bar{z} \in \operatorname{SOL}(P)$

and to assume the following relationship between the functions $f_{k}$ and $f$ :

(C4) There exists $M>0$ such that $0 \leq f\left(z_{k}\right) \leq M f_{k}\left(z_{k}\right)$ for all $k \in \mathbb{N}$.

Finally, we have also to impose the next assumption on the function $s$ :

(A1) The subdifferential operator $\partial s(\cdot)$ is bounded on bounded subsets of $H$. 
It is well known that Assumption (A1) is automatically satisfied when $H$ is a finite dimensional real Hilbert space (see, e.g. Bauschke and Borwein [10]).

Theorem 4.1 Assume that the solution set of Problem $(P)$ is nonempty and that Assumption (A1) is satisfied. If the sequences $\left\{z_{k}\right\},\left\{\lambda_{k}\right\}$ and $\left\{\rho_{k}\right\}$ generated by Algorithm 2 satisfy Conditions $(\mathrm{C} 1 \mathrm{a})$ and $(\mathrm{C} 2)-(\mathrm{C} 4)$, then the sequence $\left\{z_{k}\right\}$ converges weakly to a solution $z^{*}$ of $(P)$. Moreover, the sequence $\left\{f_{k}\left(z_{k}\right)\right\}$ converges to 0 .

Proof

(1) Let $\bar{z}$ be a solution of Problem $(P)$. Then $f(\bar{z})=0$ and $\bar{z} \in S \subset S_{k}$ for all $k$. Using Assumptions (C1a) (instead of (C1)), (C2) and (C3), we can deduce, using a proof similar to the one of Theorem 3.1, that the sequence $\left\{\left\|z_{k}-\bar{z}\right\|\right\}$ is decreasing, the sequence $\left\{z_{k}\right\}$ is bounded and the sequence $\left\{f_{k}\left(z_{k}\right)\right\}$ tends to zero. Moreover

$$
\lambda_{k}\left\|\nabla f_{k}\left(z_{k}\right)\right\|=\frac{\rho_{k} f_{k}\left(z_{k}\right)}{\left\|\nabla f_{k}\left(z_{k}\right)\right\|} \rightarrow 0 .
$$

Consequently, the sequence $\left\|z_{k+1}-z_{k}\right\| \rightarrow 0$ as $k \rightarrow \infty$. Indeed, for all $k$, we have

$$
\left\|z_{k+1}-z_{k}\right\|^{2}=\left\|\bar{z}-z_{k}\right\|^{2}-\left\|\bar{z}-z_{k+1}\right\|^{2}+2\left\langle z_{k+1}-z_{k}, z_{k+1}-\bar{z}\right\rangle
$$

and by definition of $z_{k+1}$,

$$
\left\langle z_{k+1}-\left(z_{k}-\lambda_{k} \nabla f_{k}\left(z_{k}\right)\right), \bar{z}-z_{k+1}\right\rangle \geq 0 .
$$

Hence, for all $k$, we can write, using the Cauchy-Schwarz inequality

$$
\begin{aligned}
\left\langle z_{k}-z_{k+1}, \bar{z}-z_{k+1}\right\rangle & \leq \lambda_{k}\left\langle\nabla f_{k}\left(z_{k}\right), \bar{z}-z_{k+1}\right\rangle \\
& \leq \lambda_{k}\left\|\nabla f_{k}\left(z_{k}\right)\right\|\left\|\bar{z}-z_{k+1}\right\| .
\end{aligned}
$$

Gathering (18) and (19), we deduce that for all $k$

$$
\left\|z_{k+1}-z_{k}\right\|^{2} \leq\left\|\bar{z}-z_{k}\right\|^{2}-\left\|\bar{z}-z_{k+1}\right\|^{2}+2 \lambda_{k}\left\|\nabla f_{k}\left(z_{k}\right)\right\|\left\|\bar{z}-z_{k+1}\right\| .
$$

So

$$
\left\|z_{k+1}-z_{k}\right\| \rightarrow 0 \quad \text { as } k \rightarrow \infty
$$

because the sequence $\left\{\left\|\bar{z}-z_{k}\right\|\right\}$ is convergent and $\lambda_{k}\left\|\nabla f_{k}\left(z_{k}\right)\right\| \rightarrow 0$.

(2) Let $z^{*}$ be a weak limit point of the sequence $\left\{z_{k}\right\}$. Then there exists a subsequence of $\left\{z_{k}\right\}$, denoted again $\left\{z_{k}\right\}$, that converges weakly to $z^{*}$. Since, by construction, $z_{k+1} \in S_{k}$, we have

$$
s\left(z_{k}\right)+\left\langle\psi_{k}, z_{k+1}-z_{k}\right\rangle \leq 0
$$

where $\psi_{k} \in \partial s\left(z_{k}\right)$. The subdifferential operator $\partial s(\cdot)$ being bounded on bounded subsets of $H$ (in virtue of (A1)), there exists $\Psi>0$ such that $\left\|\psi_{k}\right\| \leq \Psi$ for all $k$. Then, following (20),

$$
s\left(z_{k}\right) \leq-\left\langle\psi_{k}, z_{k+1}-z_{k}\right\rangle \leq \Psi\left\|z_{k+1}-z_{k}\right\| \rightarrow 0 .
$$

So, from the weak lower semicontinuity of the mapping $s$, we obtain that

$$
s\left(z^{*}\right) \leq \liminf _{k} s\left(z_{k}\right) \leq 0 .
$$


But this means that $z^{*} \in S$.

(3) Since $f_{k}\left(z_{k}\right) \rightarrow 0$, we obtain from Condition (C4) that $f\left(z_{k}\right) \rightarrow 0$. So, the function $f$ being convex and l.s.c. (in the strong topology) is weakly l.s.c. and

$$
0 \leq f\left(z^{*}\right) \leq \liminf _{k} f\left(z_{k}\right)=0 .
$$

Since $z^{*} \in S$, this implies that $z^{*}$ is a solution of Problem $(P)$.

Finally, using again the proof of Theorem 3.1, we can conclude that the weak limit of the sequence $\left\{z_{k}\right\}$ is unique. So the whole sequence $\left\{z_{k}\right\}$ converges weakly to $z^{*}$.

Now we examine Problem $(S E P)$ where $f(x, y)=\frac{1}{2}\|A x-B y\|^{2}$ for every $(x, y) \in$ $C \times Q$. In this case, the sets $C$ and $Q$ have a particular structure of the form

$$
C=\left\{x \in H_{1} \mid c(x) \leq 0\right\} \quad \text { and } \quad Q=\left\{y \in H_{2} \mid q(y) \leq 0\right\}
$$

where the functions $c: H_{1} \rightarrow \mathbb{R}$ and $q: H_{2} \rightarrow \mathbb{R}$ are convex and subdifferentiable.

Then the corresponding relaxed sets at iteration $k$ are defined by

$$
C_{k}=\left\{x \in H_{1} \mid c\left(x_{k}\right)+\left\langle\xi^{k}, x-x_{k}\right\rangle \leq 0\right\}
$$

and

$$
Q_{k}=\left\{y \in H_{2} \mid q\left(y_{k}\right)+\left\langle\eta^{k}, y-y_{k}\right\rangle \leq 0\right\}
$$

where $\xi^{k} \in \partial c\left(x_{k}\right)$ and $\eta^{k} \in \partial q\left(y_{k}\right)$. We have immediately that $C \subset C_{k}$ and $Q \subset Q_{k}$ for all $k$. To make the link with Algorithm 2, we define the function $s: H_{1} \times H_{2} \rightarrow \mathbb{R}$ by

$$
s(z)=s(x, y)=c(x)+q(y) .
$$

Then $C \times Q \subset S$ where $S=\left\{z \in H_{1} \times H_{2} \mid s(z) \leq 0\right\}$. Furthermore, it is easy to see that $\partial s(x, y)=\partial c(x) \times \partial q(y)$ for all $x \in H_{1}$ and $y \in H_{2}$. Here we also define the sequence $\left\{f_{k}\right\}$ by $f_{k}=f$ for all $k$. So each function $f_{k}$ is nonnegative, convex and of class $C^{1}$, and Condition (C4) is satisfied with $M=1$. In this case, Algorithm 2 can be rewritten as

\section{ALGORITHM 2-SEP}

Step 1. Let $\left(x_{0}, y_{0}\right) \in C \times Q$. Set $k=0$.

Step 2. If $A^{*}\left(A x_{k}-B y_{k}\right)=B^{*}\left(A x_{k}-B y_{k}\right)=0, \operatorname{STOP}\left(\left(x_{k}, y_{k}\right)\right.$ is a solution to Problem $(S E P))$. Otherwise, go to Step 3.

Step 3. Compute the next iterate $\left(x_{k+1}, y_{k+1}\right)$ as follows:

$$
\left\{\begin{array}{l}
x_{k+1}=P_{C_{k}}\left(x_{k}-\lambda_{k} A^{*}\left(A x_{k}-B y_{k}\right)\right) \\
y_{k+1}=P_{Q_{k}}\left(y_{k}+\lambda_{k} B^{*}\left(A x_{k}-B y_{k}\right)\right)
\end{array}\right.
$$

where the stepsize $\lambda_{k}$ is chosen in such a way that

$$
\lambda_{k}=\frac{\rho_{k}\left\|A x_{k}-B y_{k}\right\|^{2}}{2\left\|A^{*}\left(A x_{k}-B y_{k}\right)\right\|^{2}+2\left\|B^{*}\left(A x_{k}-B y_{k}\right)\right\|^{2}} \quad \text { with } \rho_{k} \in(0,4) .
$$

Step 4. Set $k \rightarrow k+1$ and go to Step 2 .

As for Algorithm1-SEP, the step $\lambda_{k}$ in our algorithm can be chosen larger than the one considered in [21]. 
Since the projections onto half-spaces $C_{k}$ and $Q_{k}$ are explicit, Algorithm 2-SEP is implementable. Now we study the convergence of Algorithm 2-SEP. For this purpose, we need the following assumption

(A2) The subdifferential operators $\partial c$ and $\partial q$ are bounded on bounded subsets of $H_{1}$ and $\mathrm{H}_{2}$, respectively.

As for Assumption (A1), it is well known that Assumption (A2) is satisfied when the Hilbert spaces $H_{1}$ and $H_{2}$ are finite dimensional.

Proposition 4.2 Assume that the solution set of Problem (SEP) is nonempty and that Assumption (A2) is satisfied. If the sequence $\left\{\rho_{k}\right\}$ satisfies Condition (C3), then the sequence $\left\{\left(x_{k}, y_{k}\right)\right\}$ generated by Algorithm 2-SEP converges weakly to a solution $\left(x^{*}, y^{*}\right)$ of $(S E P)$. Moreover, the sequence $\left\{A x_{k}-B y_{k}\right\}$ converges strongly to 0 .

Proof Since $f_{k}=f$ for all $k$, Condition (C4) is obviously satisfied. Moreover, using a proof similar to that of Proposition 3.2 with $\left(x_{k}, y_{k}\right)$ belonging to $H_{1} \times H_{2}$ instead of $C \times Q$, we obtain that the sequence $\left\{z_{k}\right\}=\left\{\left(x_{k}, y_{k}\right)\right\}$ satisfies Condition (C1a) (and thus Condition $(\mathrm{C} 1))$ and the sequence $\left\{\rho_{k}\right\}$ Condition $(\mathrm{C} 2)$.

On the other hand, setting $z_{k}=\left(x_{k}, y_{k}\right)$ and replacing $S$ by $C \times Q$ and $S_{k}$ by $C_{k} \times Q_{k}$ in the first part of the proof of Theorem 4.1, we obtain that the sequence $\left\{z_{k}\right\}$ is bounded, the sequences $\left\{f_{k}\left(z_{k}\right)\right\}$ and $\left\{\left\|z_{k+1}-z_{k}\right\|\right\}$ tend to zero. Consequently

$$
\left\|x_{k+1}-x_{k}\right\| \rightarrow 0 \text { and }\left\|y_{k+1}-y_{k}\right\| \rightarrow 0 .
$$

Let $z^{*}=\left(x^{*}, y^{*}\right)$ be a weak limit point of the sequence $\left\{z_{k}\right\}=\left\{\left(x_{k}, y_{k}\right)\right\}$. Then the second part of the proof of Theorem 4.1 may be used twice with $z_{k}$ replaced successively by $x_{k}$ and $y_{k}$ to get first that $x^{*} \in C$ and after that $y^{*} \in Q$. The third part of the proof of Theorem 4.1 gives directly that $f\left(x^{*}, y^{*}\right)=f\left(z^{*}\right)=0$ because $f\left(z_{k}\right)=f_{k}\left(z_{k}\right) \rightarrow 0$. Since $\left(x^{*}, y^{*}\right) \in C \times Q$, this implies that $\left(x^{*}, y^{*}\right)$ is a solution to Problem $(S E P)$. The rest of the proof follows directly from the end of the proof of Theorem 4.1.

To finish this section, we consider the Split Feasibility Problem:

(SFP) Find $x^{*} \in C$ such that $A x^{*} \in Q$.

Here the operator $A: H_{1} \rightarrow H_{2}$ is linear and bounded, and the subsets $C$ and $Q$ are defined as

$$
C=\left\{x \in H_{1} \mid c(x) \leq 0\right\} \quad \text { and } \quad Q=\left\{y \in H_{2} \mid q(y) \leq 0\right\}
$$

where the functions $c: H_{1} \rightarrow \mathbb{R}$ and $q: H_{2} \rightarrow \mathbb{R}$ are convex and subdifferentiable.

Then the corresponding relaxed sets at iteration $k$ are defined by

$$
C_{k}=\left\{x \in H_{1} \mid c\left(x_{k}\right)+\left\langle\xi^{k}, x-x_{k}\right\rangle \leq 0\right\}
$$

and

$$
Q_{k}=\left\{y \in H_{2} \mid q\left(A x_{k}\right)+\left\langle\eta^{k}, y-A x_{k}\right\rangle \leq 0\right\}
$$

where $\xi^{k} \in \partial c\left(x_{k}\right)$ and $\eta^{k} \in \partial q\left(A x_{k}\right)$. 
For all $k$ we define the functions $f_{k}: H_{1} \rightarrow \mathbb{R}$ by

$$
f_{k}(x)=\frac{1}{2}\left\|\left(I-P_{Q_{k}}\right) A x\right\|^{2} \quad \text { for every } x \in H_{1} .
$$

In this case, Algorithm 2 coincides with Algorithm 4.1 in López et al. [13] and takes the following form:

\section{ALGORITHM 2-SFP}

Step 1. Let $x_{0} \in C$. Set $k=0$.

Step 2. If $\left(I-P_{Q_{k}}\right) A x_{k}=0, \operatorname{STOP}\left(x_{k}\right.$ is a solution to Problem $\left.(S F P)\right)$.

Otherwise, go to Step 3.

Step 3. Compute the next iterate $x_{k+1}$ as follows:

$$
x_{k+1}=P_{C_{k}}\left(x_{k}-\lambda_{k} A^{*}\left(I-P_{Q_{k}}\right) A x_{k}\right)
$$

where the stepsize $\lambda_{k}$ is chosen in such a way that

$$
\lambda_{k}=\frac{\rho_{k}\left\|\left(I-P_{Q_{k}}\right) A x_{k}\right\|^{2}}{2\left\|A^{*}\left(I-P_{Q_{k}}\right) A x_{k}\right\|^{2}} \quad \text { with } \rho_{k} \in(0,4) .
$$

Step 4. Set $k \rightarrow k+1$ and go to Step 2 .

For Problem $(S F P)$ we recover from Theorem 4.1 the following convergence result due to López et al. ([13], Theorem 4.3), where the following assumption has been used:

(A3) The subdifferential operator $\partial c$ is bounded on bounded subsets of $H_{1}$.

Proposition 4.3 Assume that the solution set of Problem (SFP) is nonempty and that Assumption (A3) is satisfied. If the sequence $\left\{\rho_{k}\right\}$ satisfies Condition (C3), then the sequence $\left\{x_{k}\right\}$ generated by Algorithm 2-SFP converges weakly to a solution $x^{*}$ of $(S F P)$. Moreover, the sequence $\left\{\left(I-P_{Q}\right) A x_{k}\right\}$ converges strongly to 0 .

Proof In order to apply Theorem 4.1, we have to prove that Conditions (C1a), (C2) and (C4) are satisfied. First we observe that Conditions (C1a) and (C2) can be proven exactly as in the proof of Proposition 3.3. Consequently, for every $\bar{x} \in S$, we can write as in Theorem 3.1(a) that for all $k$

$$
\left\|x_{k+1}-\bar{x}\right\|^{2} \leq\left\|x_{k}-\bar{x}\right\|^{2}-\rho_{k}\left(4-\rho_{k}\right) \frac{\left[f_{k}\left(x_{k}\right)\right]^{2}}{\left\|\nabla f_{k}\left(x_{k}\right)\right\|^{2}} .
$$

So the sequence $\left\{x_{k}\right\}$ is bounded. Next, the solution set of Problem (SFP) being nonempty, it is easy to see that Problem $(S F P)$ is equivalent to the problem

$$
(P) \quad \text { minimize } f(x) \text { over } x \in C
$$

where $f: H_{1} \rightarrow \mathbb{R}$ is defined by

$$
f(x)=\max \{0, q(A x)\} \quad \text { for all } x \in H_{1} .
$$


This function $f$ is weakly 1.s.c. because it is convex and 1.s.c. (in the strong topology). So, to finish the proof, it remains to show that Condition (C4) is satisfied. Since $P_{Q_{k}}\left(A x_{k}\right) \in Q_{k}$, we have, by definition of $Q_{k}$, that

$$
q\left(A x_{k}\right) \leq\left\langle\eta_{k}, A x_{k}-P_{Q_{k}}\left(A x_{k}\right)\right\rangle
$$

where $\eta_{k} \in \partial q\left(A x_{k}\right)$. On the other hand, the sequence $\left\{x_{k}\right\}$ being bounded, it follows from (A3) that the sequence $\left\{\eta_{k}\right\}$ is also bounded. So there exists $M>0$ such that $q\left(A x_{k}\right) \leq$ $M\left\|\left(I-P_{Q_{k}}\right) A x_{k}\right\|$, which implies that $f\left(x_{k}\right) \leq M f_{k}\left(x_{k}\right)$ for all $k$. So Condition (C4) is satisfied.

\section{Strong convergence of the GP method}

In this Section, we use a Halpern-type procedure to modify Algorithm 1 in Section 3 in such a way that the weak convergence of the iterates becomes strong convergence. This procedure has been introduced in Halpern [19] for finding a fixed point of a nonexpansive mapping. Let $K$ be a nonempty closed convex subset of a Hilbert space $H$ and let $T: K \rightarrow K$ be a nonexpansive mapping (i.e. $\|T x-T y\| \leq\|x-y\|$ for all $x, y \in H$ ) whose fixed point set is nonempty. Let $u \in K$ be fixed. Then Halpern's algorithm generates a sequence $\left\{x_{k}\right\}$ in $K$ via the iterative procedure

$$
x_{k+1}=\alpha_{k} u+\left(1-\alpha_{k}\right) T x_{k} \quad \forall k \in \mathbb{N}
$$

where $x_{0} \in K$ and $\left\{\alpha_{k}\right\}$ is a sequence in $(0,1)$. It was shown in Wittmann $[23,26]$ and Xu [23] that if $\left\{\alpha_{k}\right\}$ satisfies the following conditions

$$
\begin{aligned}
& \lim _{k \rightarrow \infty} \alpha_{k}=0 \quad \text { and } \quad \sum_{k=0}^{\infty} \alpha_{k}=\infty \\
& \text { either } \quad \sum_{k=0}^{\infty}\left|\alpha_{k+1}-\alpha_{k}\right|<\infty \quad \text { or } \quad \lim _{k \rightarrow \infty} \frac{\alpha_{k+1}}{\alpha_{k}}=1
\end{aligned}
$$

then the sequence $\left\{x_{k}\right\}$ generated by Halpern's algorithm converges strongly to the fixed point of $T$ nearest to $u$. In [27], Suzuki used an explicit iteration similar to (24) and proved the strong convergence of the sequence $\left\{x_{k}\right\}$ under the only Assumption (B1).

In this section, our aim is to adapt Halpern's procedure for solving Problem $(P)$. Let us recall that this problem consists in minimizing a nonnegative convex function $f: H \rightarrow \mathbb{R}$ of class $C^{1}$ onto a nonempty closed convex subset $S$ of $H$. We assume that this problem has a solution $z^{*}$ such that $f\left(z^{*}\right)=0$. Furthermore, we want the sequence generated by our algorithm to be strongly convergent to the projection of a fixed element $w \in S$ onto the solution set, and that under the Assumption (B1). In that purpose we consider the following algorithm

\section{ALGORITHM 3}

Step 1. Choose $w \in S$. Let $z_{0} \in S$. Set $k=0$.

Step 2. If $\nabla f\left(z_{k}\right)=0, \operatorname{STOP}\left(z_{k} \in S O L(P)\right)$. Otherwise, go to Step 3.

Step 3. Compute the next iterate as follows:

$$
z_{k+1}=\alpha_{k} w+\left(1-\alpha_{k}\right) P_{S}\left(z_{k}-\lambda_{k} \nabla f\left(z_{k}\right)\right)
$$


where $\alpha_{k} \in(0,1)$ and the stepsize $\lambda_{k}$ is chosen in such a way that

$$
\lambda_{k}=\frac{\rho_{k} f\left(z_{k}\right)}{\left\|\nabla f\left(z_{k}\right)\right\|^{2}} \quad \text { with } \rho_{k} \in(0,4) .
$$

Step 4. Set $k \rightarrow k+1$ and go to Step 2 .

THeOREM 5.1 Assume that the solution set $\operatorname{SOL}(P)$ of Problem $(P)$ is nonempty and Conditions $(\mathrm{C} 1)-(\mathrm{C} 3)$, and $(\mathrm{B} 1)$ are satisfied. Then the sequence $\left\{z_{k}\right\}$ generated by Algorithm 3 is contained in $S$ and converges strongly to the solution $z^{*}$ of Problem $(P)$ which is the projection of $w$ onto $\operatorname{SOL}(P)$.

Proof Let $z^{*}=P_{S O L(P)} w$. By construction, the sequence $\left\{z_{k}\right\}$ is contained in the feasible set $S$. Let us define $w_{k}=P_{S}\left(z_{k}-\lambda_{k} \nabla f\left(z_{k}\right)\right)$ for all $k$. Then, Conditions (C1)-(C3) being satisfied, it follows from Theorem 3.1(a) that for all $k$

$$
\begin{aligned}
\left\|w_{k}-z^{*}\right\|^{2} & \leq\left\|z_{k}-z^{*}\right\|^{2}-\rho_{k}\left(4-\rho_{k}\right) \frac{\left[f\left(z_{k}\right)\right]^{2}}{\| \nabla f\left(\left(z_{k}\right) \|^{2}\right.} \\
& \leq\left\|z_{k}-z^{*}\right\|^{2} .
\end{aligned}
$$

Hence, using the convexity of the square of the norm and (28), we obtain for all $k$

$$
\begin{aligned}
\left\|z_{k+1}-z^{*}\right\|^{2} & =\left\|\alpha_{k}\left(w-z^{*}\right)+\left(1-\alpha_{k}\right)\left(w_{k}-z^{*}\right)\right\|^{2} \\
& \leq \alpha_{k}\left\|w-z^{*}\right\|^{2}+\left(1-\alpha_{k}\right)\left\|w_{k}-z^{*}\right\|^{2} \\
& \leq \alpha_{k}\left\|w-z^{*}\right\|^{2}+\left(1-\alpha_{k}\right)\left\|z_{k}-z^{*}\right\|^{2} \\
& \leq \max \left\{\left\|w-z^{*}\right\|^{2},\left\|z_{k}-z^{*}\right\|^{2}\right\} .
\end{aligned}
$$

So by induction, we can conclude from (29) that, for all $k$,

$$
\left\|z_{k+1}-z^{*}\right\|^{2} \leq \max \left\{\left\|w-z^{*}\right\|^{2},\left\|z_{0}-z^{*}\right\|^{2}\right\}
$$

which implies that the sequence $\left\{z_{k}\right\}$ is bounded.

On the other hand, we have successively for all $k$

$$
\begin{aligned}
\left\|z_{k+1}-z^{*}\right\|^{2}= & \left\|\alpha_{k}\left(w-z^{*}\right)+\left(1-\alpha_{k}\right)\left(w_{k}-z^{*}\right)\right\|^{2} \\
= & \alpha_{k}^{2}\left\|w-z^{*}\right\|^{2}+\left(1-\alpha_{k}\right)^{2}\left\|w_{k}-z^{*}\right\|^{2}+2 \alpha_{k}\left(1-\alpha_{k}\right)\left\langle w-z^{*}, w_{k}-z^{*}\right\rangle \\
= & \alpha_{k}^{2}\left\|w-z^{*}\right\|^{2}+\left(1-\alpha_{k}\right)^{2}\left\|w_{k}-z^{*}\right\|^{2} \\
& +2 \alpha_{k}\left\langle w-z^{*},\left(1-\alpha_{k}\right)\left(w_{k}-z^{*}\right)\right\rangle \\
= & \alpha_{k}^{2}\left\|w-z^{*}\right\|^{2}+\left(1-\alpha_{k}\right)^{2}\left\|w_{k}-z^{*}\right\|^{2} \\
& +2 \alpha_{k}\left\langle w-z^{*}, \alpha_{k}\left(w-z^{*}\right)+\left(1-\alpha_{k}\right)\left(w_{k}-z^{*}\right)\right\rangle-2 \alpha_{k}^{2}\left\|w-z^{*}\right\|^{2} \\
\leq & \left(1-\alpha_{k}\right)^{2}\left\|w_{k}-z^{*}\right\|^{2}+2 \alpha_{k}\left\langle w-z^{*}, z_{k+1}-z^{*}\right\rangle \\
\leq & \left(1-\alpha_{k}\right)\left\|w_{k}-z^{*}\right\|^{2}+2 \alpha_{k}\left\langle w-z^{*}, z_{k+1}-z^{*}\right\rangle .
\end{aligned}
$$

Hence, we can deduce, using (27), that for all $k$

$$
\begin{aligned}
\left\|z_{k+1}-z^{*}\right\|^{2} \leq & \left(1-\alpha_{k}\right)\left[\left\|z_{k}-z^{*}\right\|^{2}-\rho_{k}\left(4-\rho_{k}\right) \frac{\left[f\left(z_{k}\right)\right]^{2}}{\| \nabla f\left(\left(z_{k}\right) \|^{2}\right.}\right] \\
& +2 \alpha_{k}\left\langle w-z^{*}, z_{k+1}-z^{*}\right\rangle .
\end{aligned}
$$


Since $\alpha_{k} \rightarrow 0$ and $\inf _{k} \rho_{k}\left(4-\rho_{k}\right)>0$, we may assume without loss of generality that there exists $\sigma>0$ such that $\rho_{k}\left(4-\rho_{k}\right)\left(1-\alpha_{k}\right) \geq \sigma$. As a consequence, it follows from (31) that for all $k$

$$
\begin{aligned}
& \left\|z_{k+1}-z^{*}\right\|^{2}-\left\|z_{k}-z^{*}\right\|^{2}+\alpha_{k}\left\|z_{k}-z^{*}\right\|^{2}+\frac{\sigma\left[f\left(z_{k}\right)\right]^{2}}{\left\|\nabla f\left(z_{k}\right)\right\|^{2}} \\
& \quad \leq 2 \alpha_{k}\left\langle w-z^{*}, z_{k+1}-z^{*}\right\rangle .
\end{aligned}
$$

In particular, for all $k$, we obtain the next two inequalities

$$
\begin{aligned}
& \left\|z_{k+1}-z^{*}\right\|^{2} \leq\left(1-\alpha_{k}\right)\left\|z_{k}-z^{*}\right\|^{2}+\alpha_{k} \delta_{k} \\
& \left\|z_{k+1}-z^{*}\right\|^{2} \leq\left\|z_{k}-z^{*}\right\|^{2}-\eta_{k}+\alpha_{k} M
\end{aligned}
$$

where $\alpha_{k} \in(0,1), \alpha_{k} \rightarrow 0, \sum \alpha_{k}=\infty, 2\left\|w-z^{*}\right\|\left\|z_{k+1}-z^{*}\right\| \leq M$, and

$$
\eta_{k}=\frac{\sigma\left[f\left(z_{k}\right)\right]^{2}}{\left\|\nabla f\left(z_{k}\right)\right\|^{2}} \quad \text { and } \quad \delta_{k}=2\left\langle w-z^{*}, z_{k+1}-z^{*}\right\rangle .
$$

In order to apply Lemma 2.1 with $s_{k}=\left\|z_{k}-z^{*}\right\|^{2}$, it remains to prove that for any subsequence $\left\{n_{k}\right\}$ of $\{n\}$,

$$
\eta_{n_{k}} \rightarrow 0 \Rightarrow \quad \limsup _{k \rightarrow \infty} \delta_{n_{k}} \leq 0
$$

Let $\left\{n_{k}\right\}$ be a subsequence of $\{n\}$ such that $\eta_{n_{k}} \rightarrow 0$. Then, as in the proof of Theorem 3.1, we can obtain that every weak limit point of the sequence $\left\{z_{n_{k}}\right\}$ is a solution to Problem $(P)$. Since $z_{k} \in S$ and $w_{k}=P_{S}\left(z_{k}-\lambda_{k} \nabla f\left(z_{k}\right)\right)$ for all $k$, and $\eta_{n_{k}} \rightarrow 0$, we have, using successively the nonexpansiveness of the projection, the definition of $\lambda_{k}$ and the boundedness of $\rho_{k}$, that for all $\mathrm{k}$

$$
\begin{aligned}
\left\|w_{n_{k}}-z_{n_{k}}\right\| & =\left\|P_{S}\left(z_{n_{k}}-\lambda_{n_{k}} \nabla f\left(z_{n_{k}}\right)\right)-P_{S}\left(z_{n_{k}}\right)\right\| \\
& \leq \lambda_{n_{k}}\left\|\nabla f\left(z_{n_{k}}\right)\right\|=\frac{\rho_{n_{k}} f\left(z_{n_{k}}\right)}{\left\|\nabla f\left(z_{n_{k}}\right)\right\|} \rightarrow 0 .
\end{aligned}
$$

But this implies, by definition of $z_{n_{k}+1}$, that

$$
\left\|z_{n_{k}}-z_{n_{k}+1}\right\| \leq \alpha_{n_{k}}\left\|w-z_{n_{k}}\right\|+\left(1-\alpha_{n_{k}}\right)\left\|w_{n_{k}}-z_{n_{k}}\right\| \rightarrow 0
$$

because $\alpha_{k} \rightarrow 0$ and the sequence $\left\{z_{k}\right\}$ is bounded. Then, recalling that $z^{*}=P_{S O L(P)} w$, we can deduce from (35) that

$$
\begin{aligned}
\limsup _{k \rightarrow \infty} \delta_{n_{k}} & =2 \underset{k \rightarrow \infty}{\limsup }\left\langle w-z^{*}, z_{n_{k}+1}-z^{*}\right\rangle \\
& =2 \limsup _{k \rightarrow \infty}\left\langle w-z^{*}, z_{n_{k}}-z^{*}\right\rangle \\
& =2 \max _{z \in \omega_{w}\left(z_{n_{k}}\right)}\left\langle w-P_{\operatorname{SOL}(P)} w, z-P_{\operatorname{SOL}(P)} w\right\rangle \leq 0 .
\end{aligned}
$$

Applying finally Lemma 2.1, we can conclude that $\left\|z_{k}-z^{*}\right\| \rightarrow 0$. Hence the sequence $\left\{z_{k}\right\}$ converges strongly to $z^{*}$.

Now we examine successively the particular problems $(S E P)$ and $(S F P)$. Let us recall that when Problem $(S E P)$ is considered, the function $f$ is defined, for every $(x, y) \in C \times Q$, 
by $f(x, y)=\frac{1}{2}\|A x-B y\|^{2}$. Then

$$
\nabla f(x, y)=\left(A^{*}(A x-B y),-B^{*}(A x-B y)\right)
$$

for every $(x, y) \in C \times Q$. In this case, Algorithm 3 takes the following form:

\section{ALGORITHM 3-SEP}

Step 1. Choose $(u, v) \in C \times Q$. Let $\left(x_{0}, y_{0}\right) \in C \times Q$. Set $k=0$.

Step 2. If $A^{*}\left(A x_{k}-B y_{k}\right)=-B^{*}\left(A x_{k}-B y_{k}\right)=0, \operatorname{STOP}\left(\left(x_{k}, y_{k}\right)\right.$ is a solution to Problem $(S E P))$. Otherwise, go to Step 3.

Step 3. Compute the next iterate $\left(x_{k+1}, y_{k+1}\right)$ as follows:

$$
\left\{\begin{array}{l}
x_{k+1}=\alpha_{k} u+\left(1-\alpha_{k}\right) P_{C}\left(x_{k}-\lambda_{k} A^{*}\left(A x_{k}-B y_{k}\right)\right) \\
y_{k+1}=\alpha_{k} v+\left(1-\alpha_{k}\right) P_{Q}\left(y_{k}+\lambda_{k} B^{*}\left(A x_{k}-B y_{k}\right)\right)
\end{array}\right.
$$

where $\alpha_{k} \in(0,1)$ and the stepsize $\lambda_{k}$ is chosen in such a way that

$$
\lambda_{k}=\frac{\rho_{k}\left\|A x_{k}-B y_{k}\right\|^{2}}{2\left\|A^{*}\left(A x_{k}-B y_{k}\right)\right\|^{2}+2\left\|B^{*}\left(A x_{k}-B y_{k}\right)\right\|^{2}} \quad \text { with } \rho_{k} \in(0,4) .
$$

Step 4. Set $k \rightarrow k+1$ and go to Step 2 .

As for Algorithm1-SEP, the step $\lambda_{k}$ in our algorithm can be chosen larger than the one considered in [21].

When Problem $(S F P)$ is considered, the function $f$ is defined, for every $x \in C$, by $f(x)=\frac{1}{2}\left\|\left(I-P_{Q}\right) A x\right\|^{2}$. Then $\nabla f(x)=A^{*}\left(I-P_{Q}\right) A x$ for every $x \in C$. In that case, Algorithm 3 coincides with Algorithm 5.1 in López et al. [13] and takes the following form:

\section{ALGORITHM 3-SFP}

Step 1. Choose $u \in C$. Let $x_{0} \in C$. Set $k=0$.

Step 2. If $\left(I-P_{Q}\right) A x_{k}=0, \operatorname{STOP}\left(x_{k}\right.$ is a solution to Problem $\left.(S F P)\right)$.

Otherwise, go to Step 3.

Step 3. Compute the next iterate $x_{k+1}$ as follows:

$$
x_{k+1}=\alpha_{k} u+\left(1-\alpha_{k}\right) P_{C}\left(x_{k}-\lambda_{k} A^{*}\left(I-P_{Q}\right) A x_{k}\right)
$$

where $\alpha_{k} \in(0,1)$ and the stepsize $\lambda_{k}$ is chosen in such a way that

$$
\lambda_{k}=\frac{\rho_{k}\left\|\left(I-P_{Q}\right) A x_{k}\right\|^{2}}{2\left\|A^{*}\left(I-P_{Q}\right) A x_{k}\right\|^{2}} \quad \text { with } \rho_{k} \in(0,4) .
$$

Step 4. Set $k \rightarrow k+1$ and go to Step 2 .

Remark 1 Very recently, He and Zhao [28] proposed a Halpern-type algorithm to solve the Split Feasibility Problem (SFP) based on the stepsize given by López et al. [13]. Their algorithm is similar to our Algorithm 3-SFP above, but their approach and proof are quite different from ours.

Proposition 5.2 Assume that the solution set SOL(SEP) of Problem(SEP) is nonempty and that Conditions (C3) and (B1) are satisfied. Then Conditions (C1) and (C2) hold and 
the sequence $\left\{\left(x_{k}, y_{k}\right)\right\}$ generated by Algorithm 3-SEP converges strongly to a solution $\left(x^{*}, y^{*}\right)$ of Problem (SE P). This solution is the projection of $(u, v)$ onto $S O L(S E P)$.

For Problem (SFP) we recover from Theorem 5.1 the following convergence result due to López et al. ([13], Theorem 5.2).

Proposition 5.3 Assume that the solution set SOL (SFP) of Problem (SFP) is nonempty and that Conditions (C3) and (B1) are satisfied. Then Conditions $(\mathrm{C} 1)$ and $(\mathrm{C} 2)$ hold and the sequence $\left\{x_{k}\right\}$ generated by Algorithm 3-SF P converges strongly to a solution $x^{*}$ of Problem (SF P). This solution is the projection of u onto SOL (SFP).

Remark 2 A relaxed version of Algorithms 3, 3-SEP and 3-SFP can be obtained when the feasible sets $S, C$ and $Q$ are described by means of convex inequalities. The procedure is the same as the one used in Section 4 to derive Algorithms 2, 2-SEP and 2-SFP from Algorithms 1, 1-SEP and 1-SFP, respectively. At each iteration, the sets $S, C$ and $Q$ are approximated by half-spaces $S_{k}, C_{k}$ and $Q_{k}$ making the projections easy to compute.

\section{Conclusions}

A general Gradient Projection Method has been introduced for minimizing a convex function of class $C^{1}$ onto a convex constraint set. When this set is described by convex inequalities, a relaxation was proposed to make explicit the computation of the projections. In a first part, conditions were given for obtaining a weak convergence theorem and after, it was proven that these conditions are satisfied for the particular problems (SEP) and (SFP). In a second part, the Gradient Projection Method has been combined with a viscosity procedure to get a strong convergence theorem. Finally, let us mention that encouraging numerical results have been displayed in [21] where the behaviour of Algorithm 1-SEP has been compared with the classical projection algorithm (2) and the alternating CQ algorithm (3).

\section{Acknowledgements}

The authors would like to thank the three referees for their comments and suggestions on improving an earlier version of this paper. In particular, they greatly appreciated the suggestion of one of the referees to use a recent result due to He and Yang [22] for getting a more succinct proof of Theorem 5.1 .

\section{Funding}

This research is funded by the Department of Science and Technology at Ho Chi Minh City, Vietnam. Support provided by the Institute for Computational Science and Technology at Ho Chi Minh City (ICST) is gratefully acknowledged.

\section{References}

[1] Byrne Ch, Moudafi A. Extensions of the CQ algorithms for the split feasibility and split equality problems. Working paper UAG 2013-01.

[2] Moudafi A. Alternating CQ-algorithms for convex feasibility and split fixed-point problems. J. Nonlinear Convex Anal. 2014;15:809-818.

[3] Moudafi A. A relaxed alternating CQ-algorithms for convex feasibility problems. Nonlinear Anal. 2013;79:117-121. 
[4] Attouch H, Bolte J, Redont P, Soubeyran A. Alternating proximal algorithms for weakly coupled minimization problems. Applications to dynamical games and PDEs. J. Convex Anal. 2008;15:485-506.

[5] Censor Y, Elfving T. A multiprojection algorithm using Bregman projections in a product space. Numer. Algorithms. 1994;8:221-239.

[6] Byrne Ch. A unified treatment of some iterative algorithms in signal processing and image reconstruction. Inverse Prob. 2004;20:103-120.

[7] Stark H, editor. Image recovery: theory and applications. San Diego (CA): Academic Press; 1987.

[8] Censor Y, Bortfeld T, Martin B, Trofimov A. A unified approach for inversion problems in intensity-modulated radiation therapy. Phys. Med. Biol. 2003;51:2353-2365.

[9] Byrne Ch. Iterative oblique projection onto convex sets and the split feasibility problem. Inverse Prob. 2002;18:441-453.

[10] Bauschke HH, Borwein JM. On projection algorithms for solving convex feasibility problems. SIAM Rev. 1996;38:367-426.

[11] López G, Martín-Márquez V, Xu HK. Iterative algorithms for the multiple-sets split feasibility problem. In: Censor Y, Jiang M, Wang G, editors. Biomedical mathematics: promising directions in imaging, therapy planning and inverse problems. Madison (WI): Medical Physics; 2009. p. 243-279.

[12] Moudafi A. A note on the split common fixed-point problem for quasi-nonexpansive operators. Nonlinear Anal. 2011;74:4083-4087.

[13] López G, Martín-Márquez V, Wang F, Xu HK. Solving the split feasibility problem without prior knowledge of matrix norms. Inverse Prob. 2012;28:085004 (18pp). doi:10.1088/0266-5611/28/8/085004.

[14] Moudafi A, Al-Shemas E. Simultaneous iterative methods for split equality problems. Trans. Math. Program. Appl. 2013;1:1-11.

[15] Yang Q. On variable-step relaxed projection algorithm for variational inequalities. J. Math. Anal. Appl. 2005;302:166-179.

[16] Polyak BT. Introduction to optimization. New York (NY): Optimization Software; 1987.

[17] Xu HK. Iterative methods for the split feasibility problem in infinite-dimensional Hilbert spaces. Inverse Prob. 2010;26:105018 (17pp).

[18] Yang Q. The relaxed CQ algorithm for solving the split feasibility problem. Inverse Prob. 2004;20:1261-1266.

[19] Halpern B. Fixed points of nonexpanding maps. Bull. Am. Math. Soc. 1967;73:591-597.

[20] Chen R, Li J, Ren Y. Regularization method for the approximate split equality problem in infinite-dimensional Hilbert spaces. Abstr. Appl. Anal. 2013; ID 813635: 5 pages.

[21] Dong Q-L, He S, Zhao J. Solving the split equality problem without prior knowledge of operator norms. Optimization. 2014. doi:10.1080/02331934.2014.895897.

[22] He S, Yang C. Solving the variational inequality problem defined on intersection of finite level sets. Abstr. Appl. Anal. 2013; Article ID 942315.

[23] Xu HK. Iterative algorithms for nonlinear operators. J. Lond. Math. Soc. 2002;66:240-256.

[24] Konnov IV. Equilibrium models and variational inequalities. Amsterdam: Elsevier; 2007.

[25] Fukushima M. A relaxed projection method for variational inequalities. Math. Program. 1986;35:58-70.

[26] Wittmann R. Approximation of fixed points of nonexpansive mappings. Arch. Math. 1992;58:486-491.

[27] Suzuki T. A sufficient and necessary condition for Halpern-type strong convergence to fixed points of nonexpansive mappings. Proc. Am. Math. Soc. 2007;135:99-106.

[28] He S, Zhao Z. Strong convergence of a relaxed CQ algorithm for the split feasibility problem. J. Inequal. Appl. 2013;2013:197 (11pp). doi:10.1186/1029-242X-2013-197. 